\title{
Germanate oxide impacts on the optical and gamma radiation shielding properties of TeO2-ZnO-Li2O glass system
}

\begin{abstract}
In this work, a series of tellurite glass combined with various concentrations of germanium oxide was fabricated according to the formula of (70-x)TeO2-xGeO2-20ZnO-10Li2O where $\mathrm{x}=5,10$, 15 and 20 mol\% via utilizing the melt-quench method for possible use in a radiation shielding applications. X-ray diffraction and Attenuated Total Reflectance Fourier Transform Infrared was employed to investigate the structure of the synthesized glasses. The density and Poisson's ratio for current samples reduced gradually from 5.221-5.008 g.cm-3 and 0.134-0.131, respectively, while the enhancement in bandgap values from 3.700-3.872 eV with addition of GeO2 is observed. The linear attenuation coefficient values at $0.015 \mathrm{MeV}$ are 230.123 and $236.832 \mathrm{~cm}-1$ for samples TG1 and TG4, respectively. Moreover, the lowest half-value layer attained via TG1 and raises from 0.0030 to $3.6684 \mathrm{~cm}$ while the highest HVL attained by TG4 and raises from 0.0029 to 3.9696 $\mathrm{cm}$.
\end{abstract}

Keyword: Tellurite glasses; Germanate; XRDFTIR; Optical absorption; Radiation shielding 